Pacific Journal of 


\section{ENUMERATION OF DOUBLY UP-DOWN PERMUTATIONS}

\section{Carlitz}

It is well known that $A(n)$, the number of up-down permutations of $\{1,2, \cdots, n\}$ satisfies

$$
\begin{gathered}
\sum_{n=0}^{\infty} A(2 n) \frac{z^{2 n}}{(2 n) !}=\sec z, \\
\sum_{n=0}^{\infty} A(2 n+1) \frac{z^{2 n+1}}{(2 n+1) !}=\tan z .
\end{gathered}
$$

In the present paper generating functions are obtained for up-down (down-up) permutations in which the peaks themselves are in an up-down configuration.

In a previous paper the writer obtained generating functions for the number of up-down (and down-up) permutations counting the rises among the "peaks".

1. Let $Z_{n}=\{1,2, \cdots, n\}$ and let $\left(a_{1}, a_{2}, \cdots, a_{n}\right)$ be an arbitrary [4, pp. 105-112] up-down permutation of $Z_{n}$. Then $\left(b_{1}, b_{2}, \cdots, b_{n}\right)$, where

$$
b_{i}=n-a_{i}+1 \quad(i=1,2, \cdots, n)
$$

is a down-up permutation and vice versa. Thus, for $n>1$, there is a one-to-one correspondence between up-down and down-up permutations so that it suffices to consider the former.

In the present paper we are concerned with up-down (and down-up) permutations of $Z_{n}$ in which it is required that the peaks themselves satisfy the up-down or down-up conditions. Thus let $\left(a_{1}, a_{2}, \cdots, a_{n}\right)$ denote an up-down permutation of $Z_{n}$ so that

$$
a_{2 k-1}<a_{2 k}, \quad a_{2 k}>a_{2 k+1} \quad(k=1,2, \cdots,[n / 2]) .
$$

Then the additional requirement is either

$$
a_{4 k-2}<a_{4 k}, \quad a_{4 k}>a_{4 k+2} \quad(k=1,2, \cdots,[n / 4])
$$

or

$$
a_{4 k-2}>a_{4 k}, \quad a_{4 k}<a_{4 k+2} \quad(k=1,2, \cdots,[n / 4]) .
$$


For example the permutation $(1,3,2,8,4,7,6,13,9,10,5,12,11)$

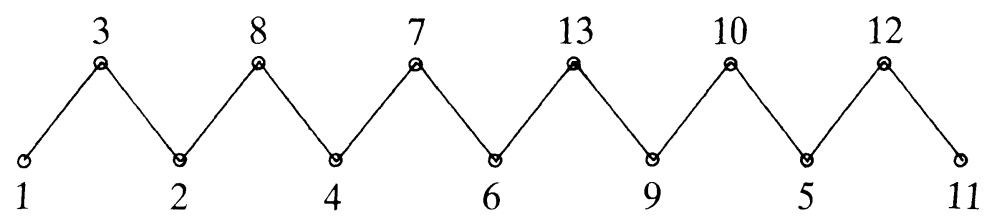

satisfies both (1.1) and (1.2).

Next let $\left(a_{1}, a_{2}, \cdots, a_{n}\right)$ be a down-up permutation of $Z_{n}$ so that

$$
a_{2 k-1}>a_{2 k}, \quad a_{2 k}<a_{2 k+1} \quad(k=1,2, \cdots,[n / 2]) .
$$

Then the additional requirement is either

$$
a_{4 k-3}>a_{4 k-1}, \quad a_{4 k-1}<a_{4 k+1} \quad(k=1,2, \cdots,[n / 4])
$$

or

$$
a_{4 k-3}<a_{4 k-1}, \quad a_{4 k-1}<a_{4 k+1} \quad(k=1,2, \cdots,[n / 4]) .
$$

Thus there are four possibilities, namely
I. (1.1) and (1.2),
II. (1.1) and (1.3),
III. (1.4) and (1.2)',
IV. (1.4) and (1.3)'.

There are various relations between these varieties of permutations; however they depend upon the residue of $n(\bmod 4)$.

In order to derive generating functions it will be convenient to define the following enumerants. Let $A_{R F}(n)$ denote the number of up-down permutations of $Z_{n}$ such that the peaks

$$
\left(a_{2}, a_{4}, \cdots, a_{2[n / 2]}\right)
$$

begin with a rise, $a_{2}<a_{4}$, and end with a fall. Thus for this case it is necessary that $n \equiv 2$ or $3(\bmod 4)$.

We define $A_{R R}(n), A_{F R}(n), A_{F F}(n)$ in a similar way. Note that for $R R, n \equiv 0$ or $1(\bmod 4)$; for $F R, n \equiv 2$ or $3(\bmod 4)$; for $F F, n \equiv 0$ or 1 $(\bmod 4)$.

We also define $C_{R F}(n), C_{R R}(n), C_{F R}(n), C_{F F}(n)$ in an analogous manner for down-up permutations. Then for $R F, n \equiv 1$ or $2(\bmod 4)$, for $R R, n \equiv 0$ or $3(\bmod 4)$, for $F R, n \equiv 1$ or $2(\bmod 4)$, for $F F, n \equiv 0$ or 3 $(\bmod 4)$.

We shall accordingly consider the following enumerants: 


$$
\begin{aligned}
& \begin{cases}A_{R F}(4 n+3), & A_{R F}(4 n+2) \\
A_{R R}(4 n+1), & A_{R R}(4 n) \\
A_{F R}(4 n+3), & A_{F R}(4 n+2) \\
A_{F F}(4 n+1), & A_{F F}(4 n)\end{cases} \\
& \begin{cases}C_{R F}(4 n+1), & C_{R F}(4 n+2) \\
C_{R R}(4 n+3), & C_{R R}(4 n) \\
C_{F R}(4 n+1), & C_{F R}(4 n+2) \\
C_{F F}(4 n+3), & C_{F F}(4 n) .\end{cases}
\end{aligned}
$$

Reading a permutation both from left to right and from right to left, we get the following relations connecting the enumerants.

$$
\left\{\begin{array}{l}
A_{R R}(4 n+1)=A_{F F}(4 n+1) \\
A_{R R}(4 n)=C_{F F}(4 n) \\
A_{F F}(4 n)=C_{R R}(4 n) \\
A_{R F}(4 n+2)=C_{R F}(4 n+2) \\
A_{F R}(4 n+2)=C_{F R}(4 n+2) \\
C_{R R}(4 n+3)=C_{F F}(4 n+3)
\end{array}\right.
$$

Put

$$
\begin{array}{ll}
y_{R F}(x)=\sum_{n=0}^{\infty} A_{R F}(4 n+3) \frac{x^{4 n+3}}{(4 n+3) !} & \left(A_{R F}(3)=2\right) \\
y_{R R}(x)=\sum_{n=0}^{\infty} A_{R R}(4 n+1) \frac{x^{4 n+1}}{(4 n+1) !} & \left(A_{R R}(1)=1\right) \\
y_{F R}(x)=\sum_{n=0}^{\infty} A_{F R}(4 n+3) \frac{x^{4 n+3}}{(4 n+3) !} & \left(A_{F R}(3)=2\right) \\
y_{F F}(x)=\sum_{n=0}^{\infty} A_{F F}(4 n+1) \frac{x^{4 n+1}}{(4 n+1) !} & \left(A_{F F}(1)=1\right) \\
z_{R F}(x)=\sum_{n=0}^{\infty} A_{R F}(4 n+2) \frac{x^{4 n+2}}{(4 n+2) !} & \left(A_{R F}(2)=1\right) \\
z_{R R}(x)=\sum_{n=0}^{\infty} A_{R R}(4 n) \frac{x^{4 n}}{(4 n) !} & \left(A_{R R}(0)=1\right) \\
z_{F R}(x)=\sum_{n=0}^{\infty} A_{F R}(4 n+2) \frac{x^{4 n+2}}{(4 n+2) !} & \left(A_{F R}(2)=1\right) \\
z_{F F}(x)=\sum_{n=0}^{\infty} A_{F F}(4 n) \frac{x^{4 n}}{(4 n) !} & \left(A_{F F}(0)=1\right)
\end{array}
$$


(1.10)

$$
\begin{cases}\bar{y}_{R F}(x)=\sum_{n=0}^{\infty} C_{R F}(4 n+1) \frac{x^{4 n+1}}{(4 n+1) !} & \left(C_{R F}(1)=1\right) \\ \bar{y}_{R R}(x)=\sum_{n=0}^{\infty} C_{R R}(4 n+3) \frac{x^{4 n+3}}{(4 n+3) !} & \left(C_{R R}(3)=2\right) \\ \bar{y}_{F R}(x)=\sum_{n=0}^{\infty} C_{F R}(4 n+1) \frac{x^{4 n+1}}{(4 n+1) !} & \left(C_{F R}(1)=1\right) \\ \bar{y}_{F F}(x)=\sum_{n=0}^{\infty} C_{F F}(4 n+3) \frac{x^{4 n+3}}{(4 n+3) !} & \left(C_{F F}(3)=2\right) \\ \bar{z}_{R F}(x)=\sum_{n=0}^{\infty} C_{R F}(4 n+2) \frac{x^{4 n+2}}{(4 n+2) !} & \left(C_{R F}(2)=1\right) \\ \bar{z}_{R R}(x)=\sum_{n=0}^{\infty} C_{R R}(4 n) \frac{x^{4 n}}{(4 n) !} & \left(C_{R R}(0)=1\right) \\ \bar{z}_{F R}(x)=\sum_{n=0}^{\infty} C_{F R}(4 n+2) \frac{x^{4 n+2}}{(4 n+2) !} & \left(C_{F R}(2)=1\right) \\ \bar{z}_{F F}(x)=\sum_{n=0}^{\infty} C_{F F}(4 n) \frac{x^{4 n}}{(4 n) !} & \left(C_{F F}(0)=1\right) .\end{cases}
$$

In view of (1.7), we get

$$
\left\{\begin{array}{lll}
y_{R R}(x)=y_{F F}(x), & z_{R R}(x)=\bar{z}_{F F}(x), & z_{F F}(x)=\bar{z}_{R R}(x) \\
z_{R F}(x)=\bar{z}_{R F}(x), & z_{F R}(x)=\bar{z}_{F R}(x), & \bar{y}_{R R}(x)=\bar{y}_{F F}(x) .
\end{array}\right.
$$

Note that, for example, in taking

$$
A_{R F}(3)=A_{F R}(3)=2,
$$

we are listing the up-down permutation $(1,3,2)$ and $(2,3,1)$ both under $R F$ and $F R$. This is done so that the recurrences given below will be satisfied. A like remark applies in a number of other instances, as is evident from an examination of $(1.8), \ldots,(1.11)$.

In the remainder of the paper we evaluate the sixteen enumerants defined in $(1.8), \ldots,(1.11)$. For a summary of results see $\$ 6$ below.

2. Evaluation of $y_{R F}(x), y_{R R}(x), y_{F F}(x), y_{F R}(x)$. We consider first $y_{R F}(x)$. The method employed is to take a typical permutation of $Z_{n}$ and consider the effect of removing the largest element. This is indeed the method used in [1]. Clearly the element removed must be a peak. The given permutation breaks into two pieces one of which may be vacuous. Thus for $A_{R F}(4 n+3)$ we get the recurrence 


$$
A_{R F}(4 n+3)=\sum_{k=0}^{n-1}\left(\begin{array}{l}
4 n+2 \\
4 k+3
\end{array}\right) A_{R F}(4 k+3) A_{R F}(4 n-4 k-1)
$$

$$
\left(n \geqq 1, \quad A_{R F}(3)=2\right) \text {. }
$$

It follows that the generating function $y_{R F}(x)$ satisfies the differential equation

$$
y_{R F}^{\prime}(x)=y_{R F}^{2}(x)+x^{2}
$$

Now put

$$
y_{R F}(x)=-\frac{U^{\prime}(x)}{U(x)}
$$

where

$$
U(x)=\sum_{n=0}^{\infty} a_{n} \frac{x^{4 n}}{(4 n) !} \quad\left(a_{0}=1\right)
$$

Substituting (2.3) in (2.2) we get

$$
U^{\prime \prime}(x)+x^{2} U(x)=0 .
$$

This implies the recurrence

$$
a_{n+1}+(4 n+1)(4 n+2) a_{n}=0 \quad(n=0,1,2, \cdots)
$$

and therefore

$$
a_{n}=(-1)^{n} 1 \cdot 5 \cdot 9 \ldots(4 n-3) \cdot 2 \cdot 6 \cdot 10 \ldots(4 n-2) .
$$

Thus

$$
\frac{a_{n}}{(4 n) !}=\frac{(-1)^{n}}{3 \cdot 7 \cdot 11 \ldots(4 n-1) \cdot 4 \cdot 8 \cdot 12 \ldots 4 n}=\frac{(-1)^{n}}{4^{2 n}} \frac{1}{n !(3 / 4)_{n}}
$$

where

$$
(a)_{n}=a(a+1) \ldots(a+n-1) \text {. }
$$

Hence (2.4) becomes

$$
U(x)=\sum_{n=0}^{\infty} \frac{(-1)^{n}}{4^{2 n}} \frac{x^{4 n}}{n !(3 / 4)_{n}}={ }_{0} F_{1}\left(-; \frac{3}{4} ; \frac{x^{4}}{16}\right)
$$


in the notation of generalized hypergeometric functions [5, Ch. 5].

Alternatively we may write [5, p. 108]

$$
U(x)=\left(\frac{1}{2} x\right)^{\frac{1}{2}} \Gamma\left(\frac{3}{4}\right) J_{-1 / 4}\left(\frac{1}{2} x^{2}\right),
$$

where $J_{-1 / 4}(z)$ denotes the Bessel function of order $-1 / 4$.

In the next place, we have for $A_{R R}(4 n+1)$ the recurrence

$$
\begin{array}{r}
A_{R R}(4 n+1)=\sum_{k=0}^{n-1}\left(\begin{array}{c}
4 n \\
4 k+3
\end{array}\right) A_{R F}(4 k+3) A_{R R}(4 n-4 k+1) \\
\left(n>0, \quad A_{R R}(1)=1\right) .
\end{array}
$$

This gives

$$
y_{R R}^{\prime}(x)=y_{R F}(x) y_{R R}(x)+1 \text {. }
$$

Hence, by (2.3), (2.10) becomes

$$
U(x) y_{R R}^{\prime}(x)+U^{\prime}(x) y_{R R}(x)=U(x)
$$

and therefore

$$
y_{R R}(x)=\frac{1}{U(x)} \int U(x)
$$

where generally

By (1.12) this implies

$$
\int f(x)=\int_{0}^{x} f(t) d t
$$

$$
y_{F F}(x)=\frac{1}{U(x)} \int U(x) .
$$

Note that, by (2.7) and (2.12),

$$
\int U=\sum_{n=0}^{\infty} \frac{(-1)^{n}}{4^{2 n}} \frac{x^{4 n+1}}{n !(3 / 4)_{n}(4 n+1)} .
$$

The enumerant $A_{F R}(4 n+1)$ satisfies the recurrence

$$
A_{\mathrm{FR}}(4 n+3)=\sum_{k=0}^{n}\left(\begin{array}{l}
4 n+2 \\
4 k+1
\end{array}\right) A_{\mathrm{FF}}(4 k+1) A_{R R}(4 n-4 k+1)
$$

$$
\left(n \geqq 0, \quad A_{F R}(3)=2\right) .
$$


Thus

$$
y_{F R}^{\prime}(x)=y_{F F}(x) y_{R R}(x) .
$$

Hence, by (2.11) and (2.13),

$$
y_{F R}^{\prime}(x)=\left\{\frac{1}{U(x)} \int U(x)\right\}^{2} .
$$

It can be verified that

$$
U^{2}(x)=\sum_{n=0}^{\infty} \frac{(-1)^{n}}{4^{2 n}} \frac{(n+1)_{n} x^{4 n}}{n !(3 / 4)_{n}(3 / 4)_{n}}
$$

3. Evaluation of $z_{R F}(x), \quad z_{R R}(x), \quad z_{F F}(x), \quad z_{F R}(x)$. As above we have first

$$
\begin{array}{r}
A_{R F}(4 n+2)=\sum_{k=0}^{n-1}\left(\begin{array}{l}
4 n+1 \\
4 k+3
\end{array}\right) A_{R F}(4 k+3) A_{R F}(4 n-4 k-2) \\
\left(n>0, \quad A_{R F}(2)=1\right) .
\end{array}
$$

This yields

$$
z_{R F}^{\prime}(x)=y_{R F}(x) z_{R F}(x)+x
$$

Hence, by (2.3),

$$
U(x) z_{R F}^{\prime}(x)+U^{\prime}(x) z_{R F}(x)=x U(x),
$$

so that

$$
z_{R F}(x)=\frac{1}{U(x)} \int x U
$$

Note that

$$
\int x U=\sum_{n=0}^{\infty} \frac{(-1)^{n}}{4^{2 n}} \frac{x^{4 n+2}}{n !(3 / 4)_{n}(4 n+2)} .
$$

Next

$$
\begin{array}{r}
A_{R R}(4 n)=\sum\left(\begin{array}{l}
4 n-1 \\
4 k+3
\end{array}\right) A_{R F}(4 k+3) A_{R R}(4 n-4 k-4) \\
\left(n \geqq 1, \quad A_{R R}(0)=1\right) .
\end{array}
$$


Thus

$$
z_{R R}^{\prime}(x)=y_{R F}(x) z_{R R}(x)
$$

It follows that

$$
z_{R R}(x)=\frac{1}{U(x)}
$$

For $A_{\text {FF }}(4 n)$ we have

$$
A_{F F}(4 n)=\sum_{k=0}^{n-1}\left(\begin{array}{l}
4 n-1 \\
4 k+1
\end{array}\right) A_{F F}(4 k+1) A_{R F}(4 n-4 k-2)
$$

$$
\left(n>0, \quad A_{\mathrm{FF}}(0)=1\right) \text {. }
$$

This gives

$$
z_{F F}^{\prime}(x)=y_{F F}(x) z_{R F}(x) .
$$

Therefore, by (2.13) and (3.3),

$$
z_{\mathrm{FF}}^{\prime}(x)=\frac{1}{U^{2}(x)} \int U(x) \cdot \int x U(x) .
$$

As for $A_{F R}(4 n+2)$, we have

$$
\begin{array}{r}
A_{F R}(4 n+2)=\sum_{k=0}^{n}\left(\begin{array}{l}
4 n+1 \\
4 k+1
\end{array}\right) A_{F F}(4 k+1) A_{R R}(4 n-2 k) \\
\left(n \geqq 0, \quad A_{F R}(2)=1\right) .
\end{array}
$$

Thus

$$
z_{F R}^{\prime}(x)=y_{F F}(x) z_{R R}(x) .
$$

Then, by (2.13) and (3.6),

$$
z_{F R}^{\prime}(x)=\frac{1}{U^{2}(x)} \int U(x) .
$$

4. Evaluation of $\bar{y}_{R F}(x), \bar{y}_{R R}(x), \bar{y}_{F F}(x), \bar{y}_{F R}(x)$. To begin with 


$$
C_{R F}(4 n+1)=\sum_{k=0}^{n-1}\left(\begin{array}{c}
4 n \\
4 k+2
\end{array}\right) C_{R F}(4 k+2) A_{R F}(4 n-4 k-2)
$$

$$
\left(n>0, \quad C_{R F}(1)=1\right) .
$$

This gives

$$
\bar{y}_{R F}^{\prime}(x)=\bar{z}_{R F}(x) z_{R F}(x) .
$$

By (1.12), $\bar{z}_{R F}(x)=z_{R F}(x)$, so that (4.2) reduces to

$$
\bar{y}_{R F}^{\prime}(x)=\left\{z_{R F}(x)\right\}^{2} .
$$

This formula together with (3.3) determines $\bar{y}_{R F}(x)$.

Next

$$
\begin{array}{r}
C_{R R}(4 n+3)=\sum_{k=0}^{n}\left(\begin{array}{l}
4 n+2 \\
4 k+2
\end{array}\right) C_{R F}(4 k+2) A_{R R}(4 n-4 k) \\
\left(n \geqq 0, \quad C_{R R}(3)=1\right),
\end{array}
$$

so that

$$
\bar{y}_{R R}^{\prime}(x)=\bar{z}_{R F}(x) z_{R R}(x)=z_{R F}(x) z_{R R}(x)
$$

Hence

$$
\bar{y}_{R R}^{\prime}(x)=\frac{1}{U^{2}(x)} \int x U(x) .
$$

Since by $(1.12), \bar{y}_{F F}(x)=\bar{y}_{R R}(x)$, we have also

$$
\bar{y}_{F F}^{\prime}(x)=\frac{1}{U^{2}(x)} \int x U(x) .
$$

In the next place

$$
\begin{array}{r}
C_{F R}(4 n+1)=\sum_{k=0}^{n}\left(\begin{array}{c}
4 n \\
4 k
\end{array}\right) C_{F F}(4 k) A_{R R}(4 n-4 k) \\
\left(n \geqq 0, \quad C_{F R}(1)=1\right) .
\end{array}
$$

This gives

$$
\bar{y}_{F R}^{\prime}(x)=\bar{z}_{F F}(x) z_{R R}(x)=\left\{z_{R R}(x)\right\}^{2},
$$


since $\bar{z}_{F F}(x)=z_{R R}(x)$. Therefore, by (3.6),

$$
\bar{y}_{F R}^{\prime}(x)=\frac{1}{U^{2}(x)} \text {. }
$$

5. Second solution of (2.5). One solution of the differential equation

$$
w^{\prime \prime}+x^{2} w=0
$$

is given by $w_{1}=U(x)$. For a second solution we take

$$
w_{2}=U(x) V(x), \quad V(x)=\sum_{n=0}^{\infty} b_{n} \frac{x^{4 n+1}}{(4 n+1) !} \quad\left(b_{0}=1\right) .
$$

Clearly $w_{1}$ and $w_{2}$ are linearly independent. Substituting from (5.2) in (5.1) we get

$$
2 U^{\prime}(x) V^{\prime}(x)+U(x) V^{\prime \prime}(x)=0 .
$$

This gives

$$
V^{\prime}(x)=\frac{1}{U^{2}(x)} .
$$

Comparing (5.4) with (4.10), it is clear that

$$
V(x)=\bar{y}_{F R}(x),
$$

so that the second solution of (5.1) becomes

$$
w_{2}=U(x) \bar{y}_{F R}(x) .
$$

6. Summary of results.

$$
\begin{aligned}
& y_{R F}(x)=-\frac{U^{\prime}(x)}{U(x)} \\
& y_{R R}(x)=y_{F F}(x)=\frac{1}{U(x)} \int U(x) \\
& y_{F R}^{\prime}(x)=\left\{\frac{1}{U(x)} \int U(x)\right\}^{2}
\end{aligned}
$$




$$
z_{R F}(x)=\bar{z}_{R F}(x)=\frac{1}{U(x)} \int x U(x)
$$

$$
z_{R R}(x)=\bar{z}_{F F}(x)=\frac{1}{U(x)}
$$

$$
z_{\mathrm{FF}}^{\prime}(x)=\bar{z}_{R R}^{\prime}(x)=\frac{1}{U^{2}(x)} \int U(x) \cdot \int x U(x)
$$

$$
z_{F R}^{\prime}(x)=z_{F R}^{\prime}(x)=\frac{1}{U^{2}(x)} \int U(x)
$$

$$
\bar{y}_{R F}^{\prime}(x)=\left\{z_{R F}(x)\right\}^{2}
$$

$$
\bar{y}_{R R}(x)=\bar{y}_{F F}(x)=\frac{1}{U^{2}(x)} \int x U(x)
$$

$$
\bar{y}_{F R}^{\prime}(x)=\frac{1}{U^{2}(x)}
$$

$$
U(x)=\sum_{n=0}^{\infty} \frac{(-1)^{n}}{4^{2 n}} \frac{x^{4 n}}{n !(3 / 4)_{n}}
$$

$$
U^{2}(x)=\sum_{n=0}^{\infty} \frac{(-1)^{n}}{4^{2 n}} \frac{\left(n+\frac{1}{2}\right)_{n} x^{4 n}}{n !(3 / 4)_{n}(3 / 4)_{n}} .
$$

Linearly independent solutions of

$$
w^{\prime \prime}+x^{2} w=0
$$

are furnished by

$$
w_{1}=U(x), \quad w_{2}=U(x) \bar{y}_{\mathrm{FR}}(x) .
$$

7. Generalizations. We may define doubly up-down permutations as permutations $\left(a_{1}, a_{2}, \cdots, a_{n}\right)$ of $Z_{n}$ such that

$$
a_{2 k-1}<a_{2 k}, \quad a_{2 k}>a_{2 k+1} \quad(k=1,2, \cdots,[n / 2])
$$

and

$$
a_{4 k-2}<a_{4 k}, \quad a_{4 k}>a_{4 k+2} \quad(k=1,2, \cdots,[n / 4]) .
$$

Similarly we may define triply up-down as permutations that satisfy (7.1) and (7.2) and in addition

$$
a_{8 k-4}<a_{8 k}, \quad a_{8 k}>a_{8 k+4} \quad(k=1,2, \cdots,[n / 8]) .
$$


It is clear how to extend this definition to $r$-ply up-down permutations. Thus this suggests the enumeration of permutations of these types.

An extension in a different direction is the following. Let $A_{3}(n)$ denote the number of permutations of $Z_{n}$ that satisfy

$$
a_{3 k-2}<a_{3 k-1}<a_{3 k}, \quad a_{3 k}>a_{3 k+1} \quad(k=1,2, \cdots,[n / 3]) .
$$

Then, as a special case of a result proved in [2], [3], we have

$$
\sum_{n \times 0}^{\infty} A_{3}(3 n) \frac{x^{3 n}}{(3 n) !}=\left\{\sum_{n=0}^{\infty} \frac{(-1)^{n}}{(3 n) !}\right\}^{-1} \text {. }
$$

This suggests the consideration of permutations that satisfy (7.4) and in addition

$$
a_{9 k-6}<a_{9 k-3}<a_{9 k}, \quad a_{9 k}>a_{9 k+3} \quad(k=1,2, \cdots,[n / 9]) .
$$

Moreover further restrictions analogous to (7.3) can also be introduced. - However we shall not treat these extensions in the present paper.

\section{REFERENCES}

1. L. Carlitz, Enumeration of up-down permutations by number of rises, Pacific J. Math., 45 (1973), 49-58.

2. - Generating functions for a special class of permutations, Proc. Amer. Math. Soc., 47 (1975), 251-256.

3. - Permutations with prescribed pattern, Mathematische Nachrichten, 58 (1973), 31-53.

4. E. Netto, Lehrbuch der Combinatorik, Teubner, Leipzig and Berlin, 1927.

5. E. D. Rainville, Special Functions, Macmillan, New York, 1960.

Received July 6, 1976.

DUKE UNIVERSITY

DURHAM, NC 27706 



\section{Pacific Journal of Mathematics \\ Vol. 70, No. $1 \quad$ September, 1977}

William H. Barker, Noether's theorem for plane domains with hyperelliptic

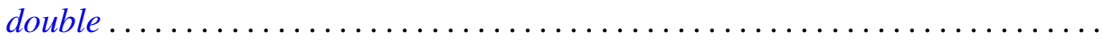

Michael James Beeson, Non-continuous dependence of surfaces of least area on the boundary curve ...................................... 11

Horst Behncke, Functions acting in weighted Orlicz algebras . . . . . . . . . . . . 19

Howard Edwin Bell, A commutativity study for periodic rings . . . . . . . . . . . 29

Peter Botta and Stephen J. Pierce, The preservers of any orthogonal group ....... 37

Douglas S. Bridges, The constructive Radon-Nikodým theorem ............. 51

James Dennis Brom, The theory of almost periodic functions in constructive

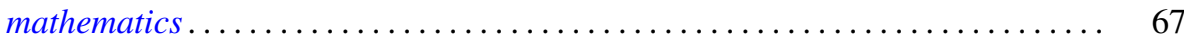

N. Burgoyne and C. Williamson, Semi-simple classes in Chevalley type groups ....

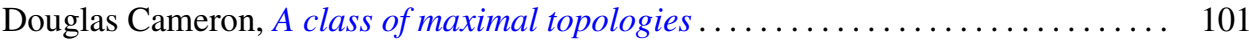

L. Carlitz, Enumeration of doubly up-down permutations . . . . . . . . . . . . . . 105

Paul Robert Chernoff, The quantum n-body problem and a theorem of

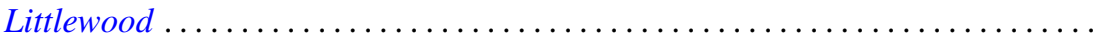

Jo-Ann Deborah Cohen, Locally bounded topologies on $F(X) \ldots \ldots \ldots \ldots \ldots \ldots$

Heinz Otto Cordes and Robert Colman McOwen, Remarks on singular elliptic

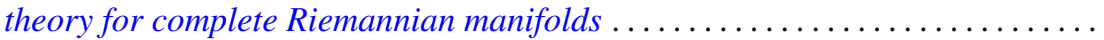

Micheal Neal Dyer, Correction to: "Rational homology and Whitehead

products"

Robert Fernholz, Factorization of Radonifying transformations

Lawrence Arthur Fialkow, A note on quasisimilarity. II ...... . .

Harvey Charles Greenwald, Lipschitz spaces of distributions on the surface of unit

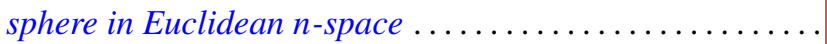

Albrecht Irle, On the measurability of conditional expectations

Tom (Roy Thomas Jr.) Jacob, Matrix transformations involving simple sequence

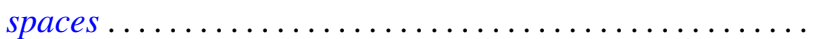

A. Katsaras, Continuous linear maps positive on increasing continuous

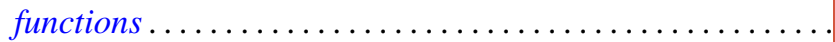

Kenneth Kunen and Judith Roitman, Attaining the spread at cardinals of cofinality

Lawrence Louis Larmore and Robert David Rigdon, Enumerating normal bundles

of immersions and embeddings of projective spaces ...... . .

Ch. G. Philos and V. A. Staïkos, Asymptotic properties of nonoscillatory solutions of differential equations with deviating argument .

Peter Michael Rosenthal and Ahmed Ramzy Sourour, On operator algebras containing cyclic Boolean algebras...

Polychronis Strantzalos, Strikt fast gleichgradig-stetige und eigentliche

Aktionen ...

Glenn Francis Webb, Exponential representation of solutions to an abstract

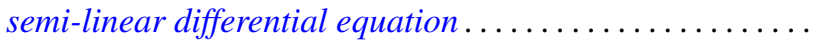

